\title{
Implicitly learned suppression of irrelevant spatial locations
}

\author{
Andrew B. Leber ${ }^{1} \cdot$ Rachael E. Gwinn ${ }^{1}$ - Yoolim Hong ${ }^{1} \cdot$ Ryan J. O'Toole ${ }^{2}$
}

Published online: 25 May 2016

(C) Psychonomic Society, Inc. 2016

\begin{abstract}
How do we ignore a salient, irrelevant stimulus whose location is predictable? A variety of studies using instructional manipulations have shown that participants possess the capacity to exert location-based suppression. However, for the visual search challenges we face in daily life, we are not often provided explicit instructions and are unlikely to consciously deliberate on what our best strategy might be. Instead, we might rely on our past experience - in the form of implicit learning - to exert strategic control. In this paper, we tested whether implicit learning could drive spatial suppression. In Experiment 1, participants searched displays in which one location contained a target, while another contained a salient distractor. An arrow cue pointed to the target location with $70 \%$ validity. Also, unbeknownst to the participants, the same arrow cue predicted the distractor location with $70 \%$ validity. Results showed facilitated RTs to the predicted target location, confirming target enhancement. Critically, distractor interference was reduced at the predicted distractor location, revealing that participants used spatial suppression. Further, we found that participants had no explicit knowledge of the cue-distractor contingencies, confirming that the learning was implicit. In Experiment 2, to seek further evidence for suppression, we modified the task to include occasional masked probes following the arrow cue; we found worse probe identification accuracy at the predicted distractor location than control locations, providing converging evidence that
\end{abstract}

Andrew B. Leber

leber.30@osu.edu

1 Department of Psychology, The Ohio State University, 225 Psychology Building, 1835 Neil Avenue, Columbus, OH 43210, USA

2 Department of Biology, Dartmouth College, Hanover, NH, USA observers spatially suppressed the predicted distractor locations. These results reveal an ecologically desirable mechanism of suppression, which functions without the need for conscious knowledge or externally guided instructions.

Keywords Attention $\cdot$ Attention capture $\cdot$ Implicit learning and memory $\cdot$ Spatial attention

Imagine watching your daughter at the playground while perusing your smartphone. Each time you glance up from your device, you initiate a visual search, prioritizing locations where you expect to find her. Your ability to do this is confirmed by decades of research on voluntary spatial attention (e.g., Eriksen \& Collins, 1969; Posner, Snyder \& Davidson, 1980). But, beyond this, can you further improve your performance by strategically ignoring locations where you are unlikely to find your daughter? For instance, can you ignore a slide she dislikes? If the slide is particularly salient, locationbased suppression would be advantageous, preventing unwanted deployments of attention.

Researchers have developed a variety of methods to study spatial suppression, including inhibition of return (IOR: Posner \& Cohen, 1984; Klein 2000), cross-trial inhibition (Leber, Lechak \& Tower-Richardi, 2013; Maljkovic \& Nakayama, 1996), antisaccade tasks (Hallett, 1978; Munoz \& Everling, 2004), delayed non-match to sample tasks (Curtis, Rao \& D'Esposito, 2004; Dhawan, Deubel, \& Jonikaitis, 2013), and explicit distractor cueing (Ruff \& Driver, 2006; Munneke, Van der Stigchel \& Theeuwes, 2008). Results from these methods have provided converging behavioral and neural evidence that individuals can indeed suppress spatial locations.

These examples rely on either recent experience (IOR, cross-trial inhibition) and/or experimenter instructions 
(antisaccade task, delayed non-match to sample, direct cueing) to establish suppression. However, the real world does not always provide these things. Consider that we sometimes wish to ignore locations we have not recently rejected. Or consider that we do not often receive instructions or consciously decide which attentional strategy to use. Even if we did, we could have poor metacognitive insight into our own strategies (Kawahara, 2010; Proulx, 2011). Thus, do we have a mechanism of spatial suppression that is both flexible and does not rely on conscious control?

Essentially, this is a question of implicit learning, or the exploitation of environmental regularities without awareness or intent (Stadler \& Frensch, 1998). Examples of implicit learning in visual processing have proliferated in recent years, such as contextual cueing (Chun \& Jiang, 1998), spatial probability learning (Miller, 1988), and visual statistical learning (Fiser \& Aslin, 2001). With respect to spatial suppression, recent studies have manipulated probability, presenting distractors more frequently in some locations than others; these manipulations yielded reduced interference from distractors in high-probable locations (Goschy, Bakos, Müller \& Zehetleitner, 2014; Kelley \& Yantis, 2009; Reder, Weber, Shang, \& Vanyukov, 2003). Such effects may have arisen from implicit learning, although they may also be explained by the accumulation of cross-trial inhibition (see e.g., Walthew \& Gilchrist, 2006). Additionally, these studies focused suppression on a single location, but can implicit learning drive flexible, trial-by-trial adjustments in suppression? The present study suggests the answer is yes, providing novel evidence that implicit learning indeed does drive flexible spatial suppression.

\section{Experiment 1}

We began by adapting the procedure of Folk, Remington \& Johnston (1992), in which participants searched four-item displays for color-defined targets while trying to ignore salient distractors appearing prior to the target. We also added Posner cueing (Posner et al., 1980), using a central arrow to predict the target location. Critically, unbeknownst to the participants, we also used this arrow to predict the distractor location. For instance, for one participant, an arrow cue pointing to the upper left predicted the target location with $70 \%$ validity while also predicting a distractor at the bottom right, with $70 \%$ validity. Each arrow direction predicted a unique distractor location.

If participants could implicitly learn the cue-distractor relationship, and if they could use this information to flexibly suppress changing distractor locations from trial to trial, we predicted reduced distractor interference on trials in which the cue-distractor relationship was valid vs. invalid. Alternatively, if individuals could not learn and implement flexible trial-by- trial suppression, we predicted no modulation of performance depending on the cue-distractor relationship.

\section{Method}

\section{Participants}

From a pilot experiment, we deemed 24 participants were needed to obtain power of 0.8 for our critical distractor validity analysis. We ran 26 participants (17 female; mean age = 20.7 years), for course credit or monetary compensation (US $\$ 10 / \mathrm{h}$ ). All participants had self-reported normal or correctedto-normal visual acuity and color vision. We obtained informed consent from all participants, and the protocols were approved by the Behavioral and Social Sciences Institutional Review Board at Ohio State. The experiment took 1.5-2 h.

\section{Apparatus and stimuli}

Stimuli were generated with an Apple computer running MATLAB (Mathworks, Natick, MA), with the Psychophysics Toolbox (Brainard, 1997; Pelli, 1997), and were presented on a 24-inch LCD monitor (refresh $=60 \mathrm{~Hz}$; resolution $=1920 \times 1280)$. Typical viewing distance was $63 \mathrm{~cm}$; head position was not fixed.

Main task trial stimuli contained fixation displays, cue/ placeholder displays, placeholder-only displays, distractor displays, and target displays, all presented on a black background (see Fig. 1). The fixation display contained a central small white circle $\left(0.15^{\circ}\right.$ visual angle in diameter $)$. The cue/ placeholder display included a central arrow cue, four

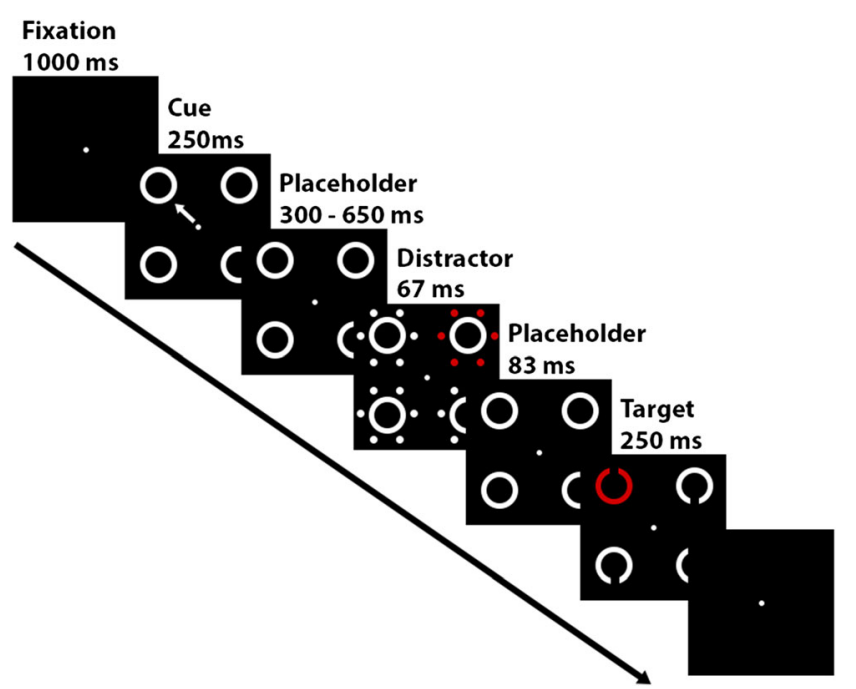

Fig. 1 Depiction of Experiment 1 trial events. Participants were instructed to search for the red circle while trying to ignore the distractor. The arrow cue predicted the target location with $70 \%$ validity, and it also - unbeknownst to participants - predicted the distractor location with $70 \%$ validity. See Materials and methods for additional details 
placeholders, and fixation. The arrow (length $=0.41^{\circ}$; width $=$ $0.31^{\circ}$; stroke $=0.05^{\circ}$ ) pointed at one of the four placeholders. Arrows were centered at $0.38^{\circ}$ eccentricity from fixation. Placeholders consisted of four gray outline circles (diameter $=1.22^{\circ} ;$ stroke $=0.10^{\circ}$; eccentricity $=2.90^{\circ}$ ), centered on the corners of an imaginary square that was centered at fixation. Placeholder-only displays contained just placeholders and fixation. Distractor displays had placeholders, fixation, and four groups of six dots, each $0.29^{\circ}$ in diameter, with one group surrounding each placeholder. Within each group, each dot was centered $0.20^{\circ}$ beyond the placeholder's outside edge, and the dots were spaced evenly, occupying the six vertices of an imaginary hexagon oriented with two horizontal sides. One group of dots was red, which we refer to collectively as the distractor. The remaining dots were gray. The search display was like the placeholder display, except one of the four outline circles, the target, was changed to red and each circle now contained a $0.29^{\circ}$ gap at either the top or bottom.

\section{Design and procedure}

Main task Trial types were produced by factorially crossing three variables, as follows:

Cued location: each of the four locations was cued equally often.

Target location: on target valid trials (70\%), the target appeared at the cued location, while on target invalid trials, it appeared equally often at the three invalid locations.

Distractor location: unbeknownst to participants, we paired each cued location with a corresponding distractor-valid location, where the distractor appeared on $70 \%$ of trials. These cue-distractor contingencies were stable across the entire experiment. On distractor-invalid trials, the distractor appeared equally often at the remaining three locations.

The cue-distractor contingencies met two constraints. First, we wanted the distractor to appear equally often at each location, so we paired each cued location with a distinct, distractor-valid location. Second, we wanted the distances between cued target and distractor locations to be matched to those that would occur by chance if the respective locations were selected randomly and independently on each trial. Chance stipulates that the cue would match the distractor location on $25 \%$ of the trials, it would be in an adjacent location on $50 \%$ of the trials, and it would be on the opposite side of the display on $25 \%$ of the trials. To match the proportions for these distances, we generated the cue-distractor contingency arrangement shown in Fig. 2. To ensure that no idiosyncrasies of this particular arrangement confounded the results, we counterbalanced it across each set of eight participants as follows: the first was assigned the arrangement shown. For the second, third, and fourth participants, we rotated the arrangement by $90^{\circ}, 180^{\circ}$, and $270^{\circ}$, respectively. Then, for the next four participants, we mirror-reversed the arrangements for participants one to four along the vertical axis.

Within each participant, the crossing of cued location, target location, and distractor location yielded one block of 400 trials. After 24 practice trials, we presented four main task blocks, yielding 1600 trials. Within blocks, the trial presentation order was randomized. Breaks were given every 100 trials.

Trials began with the fixation display (see Fig. 1). This was followed $1000 \mathrm{~ms}$ later by the cue/placeholder display, which was removed $267 \mathrm{~ms}$ later and replaced by the placeholderonly display. The placeholders remained for another $367 \mathrm{~ms}$, $517 \mathrm{~ms}$, or $667 \mathrm{~ms}$, a duration that was selected randomly on each trial. The distractor display appeared next, yielding a cuedistractor stimulus onset asynchrony (SOA) of $633 \mathrm{~ms}$, $783 \mathrm{~ms}$, or $933 \mathrm{~ms}$. The distractor display was exposed for $67 \mathrm{~ms}$ before reverting back to the placeholder display for another $83 \mathrm{~ms}$. Then, the search display appeared, yielding a distractor-target SOA of $150 \mathrm{~ms}$. Gap locations in each search item were determined randomly and independently on each trial. The search display was removed after $250 \mathrm{~ms}$, leaving the fixation display, which remained until response or $2000 \mathrm{~ms}$, whichever came first. An "ERROR" message was displayed for $1000 \mathrm{~ms}$ on non-response or incorrect-response trials.

We instructed participants to search for the red circle and report its gap location - top or bottom — by respectively using their right index finger to press " 1 " or right middle finger to press the " 2 " on a keyboard number pad. We asked participants to respond quickly and accurately, and we informed them of the irrelevant distractor and encouraged them to ignore it. Additionally, we told participants that the arrow predicted the target location but not that it predicted the distractor location.

Explicit learning assessment After the main task, we assessed participants' explicit knowledge about the cuedistractor contingencies. First, we asked, "Did you notice that it was sometimes possible to predict where the distractor items would appear?" After recording their "yes" or "no" response, we presented another message explaining that the arrow did indeed predict the distractor location, but we did not explain their specific cue-distractor contingencies. Participants then performed a generation task (Willingham, Nissen \& Bullemer, 1989), as follows. They were shown 100 consecutive trials with a cue/placeholder display. The arrow pointed equally often to each location across the trials, with presentation order randomized. The mouse cursor appeared, and participants were asked to click on the location they felt should have a distractor. They were told, "you may feel as if you are 

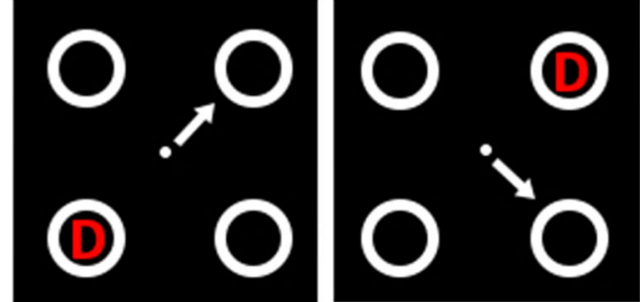

Fig. 2 Representation of cue-distractor contingencies in a sample participant. Each of the four directional arrows predicted a distinct distractor location (indicated by a $D$ ), such that the aggregate cue-

totally guessing, but if you use your 'gut' feeling, you might end up producing a correct pattern." Each display was presented until a placeholder was clicked. Then, a distractor was added at the clicked location for $500 \mathrm{~ms}$, to confirm the response. Afterwards, the display went blank for $500 \mathrm{~ms}$ before starting the next trial. No accuracy feedback was provided.

\section{Results and discussion}

Two participants were excluded for low accuracy in the main task $(71.4 \%$ and $78.9 \%)$, which fell $>2.5$ SD below the remaining participants. The final two participants were run as replacements, matching the excluded participants' cuedistractor arrangements.

\section{Overall behavior}

Our first analysis of RT and error rates was a manipulation check for both endogenous cueing and spatial capture effects (Posner et al., 1980; Folk et al., 1992; see Fig. 3).

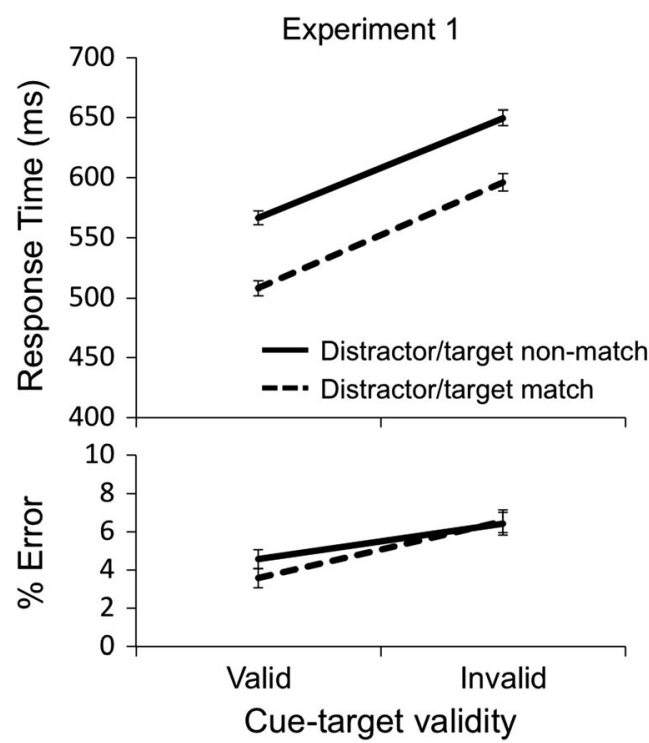

Fig. 3 Basic RTs and error rates for Experiments 1 and 2 (left and right, respectively), allowing an appraisal of Posner cueing (main effect of cuetarget validity) and attentional capture (main effect of distractor/target
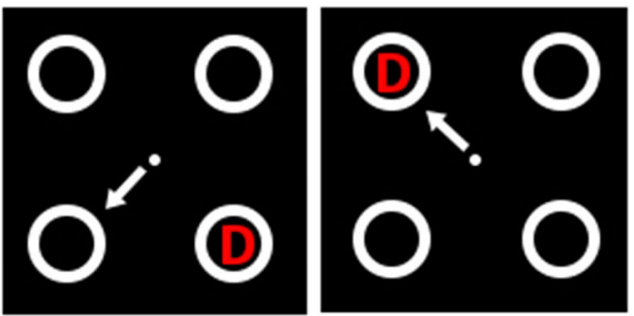

distractor distances were matched to chance and the distractor appeared equally often at each display location

For RT, we excluded error trials and trials with RTs exceeding 3 SD above the participants' means, separately for cuetarget valid and invalid conditions $(1.50 \%$ and $1.36 \%$ of correct trials, respectively). We then computed a two factor (cue validity $\times$ distractor-target location match), repeated measures ANOVA. This yielded a significant main effect of cuetarget validity, $F(1,23)=46.366, P<0.00001, \eta_{p}{ }^{2}=.668$, showing robust endogenous cueing, by which RT was faster to validly than invalidly cued targets. There was also a significant main effect of distractor-target location match, $F(1,23)=$ 89.422, $P<0.00001, \eta_{p}{ }^{2}=.795$, demonstrating robust spatial capture, by which RTs were slowed when distractors and targets were presented in different locations than same locations. The two-way interaction was not significant, $F<1$.

Error rates were entered into the same ANOVA. Results yielded a significant main effect of cue-target validity, $F(1$, 23) $=11.910, P=0.002, \eta_{p}{ }^{2}=.341$, revealing worse performance on invalid than valid trials. The main effect of distractor-target location match was not significant, $F<1$. These effects were qualified by a significant two-way

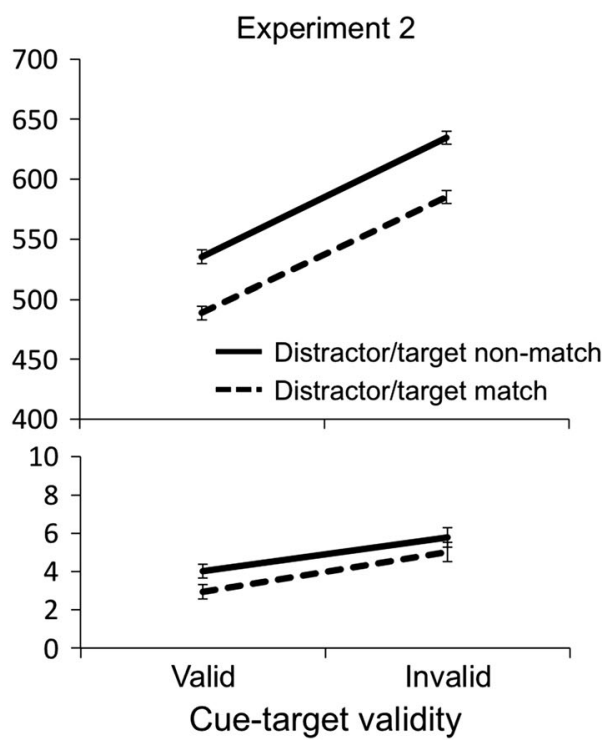

match). Error bars indicate the standard error (SE) of the difference between distractor/target match vs. non-match, separately for each level of target validity 
interaction, $F(1,23)=4.639, P=0.042, \eta_{p}^{2}=.168$, reflecting greater spatial capture on valid trials than on invalid trials.

Overall, these results confirm successful manipulations of endogenous cueing (Posner et al., 1980) and spatial capture (Folk et al., 1992).

\section{Distractor validity effects}

Next, we assessed whether and how performance depended on cue-distractor validity. We assessed this separately from the overall analysis above, for several reasons. First, we focused only on trials in which we could isolate any potential benefits of the distractor validity from effects of target validity; this led us to exclude trials in which the distractor was predicted to and/or did appear at the target location. Second, because implicit knowledge of the cue-distractor relationship might emerge over time, we analyzed the learning effect by block.

We entered RT data into a 3-factor ANOVA (distractor validity $\times$ target validity $\times$ block); means are plotted in Fig. 4. Beginning with the central test of this experiment, the main effect of distractor validity was significant, $F(1,23)=$ 15.437, $P=0.00067, \eta_{p}^{2}=.402$, reflecting faster RTs on distractor-valid than on distractor-invalid trials. This demonstrates that observers learned to ignore the distractors. The main effect of target validity was based largely on the same data as the earlier test in the overall RT analysis, so it is a nonindependent restatement of that analysis, $F(1,23)=40.102, P$ $<0.000001, \eta_{p}{ }^{2}=.636$. For the block main effect, Mauchly's test showed a violation of the sphericity assumption, so we

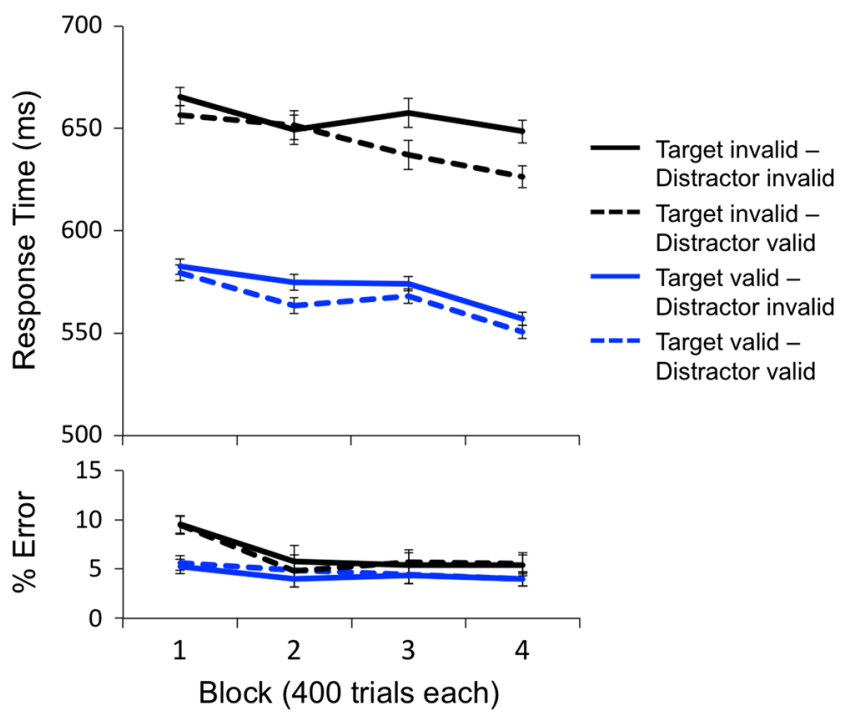

Fig. 4 Experiment $1 \mathrm{RT}$ and error rates as a function of cue-distractor validity (distractor validity), plotted separately by cue-target validity (target validity) and block. Error bars indicate the SE of the difference between distractor invalid and valid, separately for each level of target validity and block applied the Greenhouse-Geisser correction; results were nonsignificant, $F(1.55,35.65)=1.785$ (n.s.).

This distractor validity effect did not interact with target validity, $F<1$, showing that learning was equivalent whether or not the target appeared in its predicted location. Additionally, while the distractor validity effect increased numerically after the first block, it did not interact significantly with block, $F(3,69)=1.891$ (n.s.). The target validity $\times$ distractor validity interaction was not significant, $F(1,23)=$ 1.952 (n.s.), although the 3-way target validity $\times$ distractor validity $\times$ block interaction was significant, $F(2.214,48.846)$ $=3.727, P=0.029, \eta_{p}^{2}=.139$ (Greenhouse-Geisser corrected). This likely reflected a greater increase in learning across blocks in the target-invalid trials than the target-valid trials.

The error rates were broadly consistent with the RT results. We observed a main effect of target validity (restating the earlier test on overall behavior), $F(1,23)=$ 5.287, $P=0.031, \eta_{p}{ }^{2}=.187$. No other main effects or interactions reached significance, for which all $F$ values < 1 , except the main effect of block, $F(1.609,37.014)=$ 1.944 , and the target validity $\times$ block interaction, $F(1.900,43.711)=1.399$ (both Greenhouse-Geisser corrected).

\section{Explicit learning assessment}

When asked if they noticed that the distractor location's predictability, 9 participants said yes and 15 said no. However, T-tests showed that neither of these groups guessed the location reliably above chance during the generation task, $t(8)=1.584$ (n.s.), and $t(14)=0.575$ (n.s.), respectively (Fig. 5). Clearly, participants did not explicitly learn the relationship between the arrow cue and the predicted distractor location.

\section{Experiment 2}

The distractor validity effect provides evidence that individuals learned to suppress implicitly cued distractor locations. However, one alternative is that participants did not use suppression but rather slowed responses on distractorinvalid trials due to an expectancy violation. A related alternative is that participants strategically enhanced the predicted distractor location, to process and subsequently overcome its interference more rapidly (see, e.g., Moher \& Egeth, 2012; Tsal \& Makovski, 2006). Experiment 2 tested these possibilities by introducing occasional masked probes after the cue at one of the four display locations. If participants suppress the predicted distractor location, then their probe identification accuracy should be poorer at predicted vs. non-predicted distractor locations. In contrast, 


\section{Experiment 1}

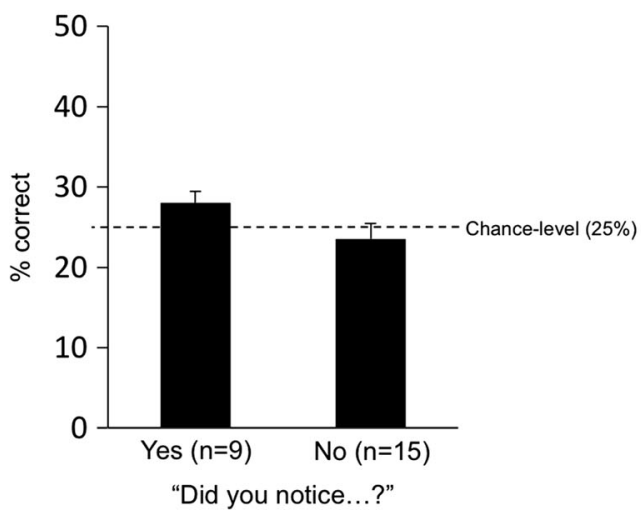

Fig. 5 Generation task performance in Experiments 1 and 2 (left and right, respectively), plotted separately for those who reported noticing it was sometimes possible to predict the distractor location during the main

by an expectancy violation account, we would not hypothesize poorer probe accuracy at the predicted distractor location. Moreover, by a strategic enhancement account, which posits greater attention to the predicted distractor location, we would hypothesize greater accuracy to probes at the predicted vs. non-predicted distractor locations.

\section{Method}

We ran 25 new participants ( 17 female; mean age $=$ 21.4 years), in an experiment based on Experiment 1 and modified as follows.

The Main Task was now comprised of both Search and Masked Probe trials, which were presented on $80 \%$ and $20 \%$ of trials, respectively. The trial types were presented in random order within blocks, with the constraint that no more than two probe trials could occur in a row.

Search trials were based on those described in Experiment 1. As before, the cue-target validity was $70 \%$, although now the cue-distractor validity was increased to $100 \%$. We made this change because including probe trials (explained below) meant that distractors did not always follow cues, and we did not want to diminish cue-distractor learning. We consequently could not analyze distractor validity and planned to focus solely on the probe trials to assess learning and distractor suppression.

Masked Probe trials began with the same fixation, placeholder, and cue displays as the search trials (see Fig. 6). Then, a red or vertical horizontal probe bar (length $=0.48^{\circ}$; stroke $=$ $0.10^{\circ}$ ), centered inside one of the placeholders, was presented for a variable duration that was adjusted adaptively. It was followed by a 100 -ms mask of superimposed horizontal and vertical red bars. Then, a prompt appeared (until response or $6000 \mathrm{~ms}$, whichever came first), requesting observers to report if the probe was vertical or horizontal, by pressing the ' 1 ' or
Experiment 2

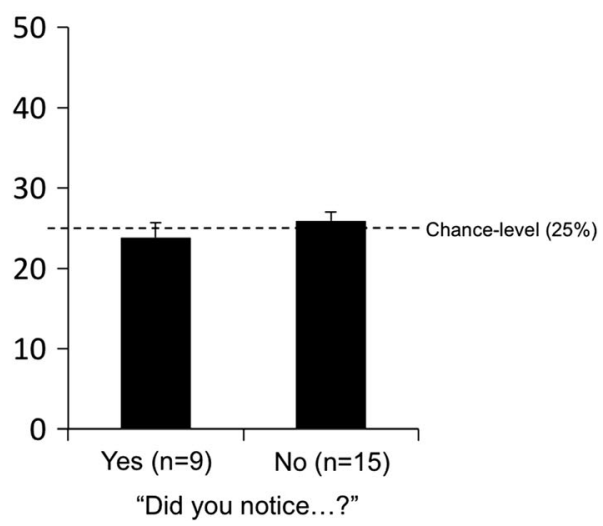

experiment. The horizontal dashed line marks chance accuracy. Error bars indicate SE of the mean

'2' response buttons, respectively. Probes appeared equally often at each location, independent of cue-arrow direction.

Participants practiced the search task for 24 trials, and then the probe task for 24 trials. During probe practice, the initial probe exposure was set to $150 \mathrm{~ms}$ and then titrated based on performance to a value ranging between $50 \mathrm{~ms}$ and $150 \mathrm{~ms}$. Arrow cues were omitted during probe practice. After practice, participants completed two consecutive blocks of 800 trials each (640 search and 160 probe), with breaks every 100 trials. At each break, we adjusted the probe exposure duration up or down by $16.7 \mathrm{~ms}$ if accuracy fell outside of 65-85\%. After the main task, we administered the Explicit Learning Assessment used in Experiment 1.

\section{Results and discussion}

One participant was excluded for low accuracy in the main task search trials $(83.4 \%$ ), which was $>2.5$ SD lower than the remaining participants; the last participant was run as a replacement.

\section{Search trials}

For RT, we carried out the same ANOVA assessing endogenous cueing and attentional capture as in Experiment 1 (see Fig. 3). Results once again showed a main effect of cue validity, $F(1,23)=75.544, P<0.000001, \eta_{p}{ }^{2}=.767$, a main effect of distractor-target location match, $F(1,23)=96.293, P<$ $0.000001, \eta_{p}{ }^{2}=.807$, and no significant interaction, $F<1$. The same analysis on error rates mirrored the RT results, yielding a main effect of cue validity, $F(1,23)=15.037, P=0.001$, $\eta_{p}^{2}=.395$, and a main effect of distractor-target match, $F(1$, 23) $=8.576, P<0.008, \eta_{p}{ }^{2}=.272$. The interaction was not significant, $F<1$. 


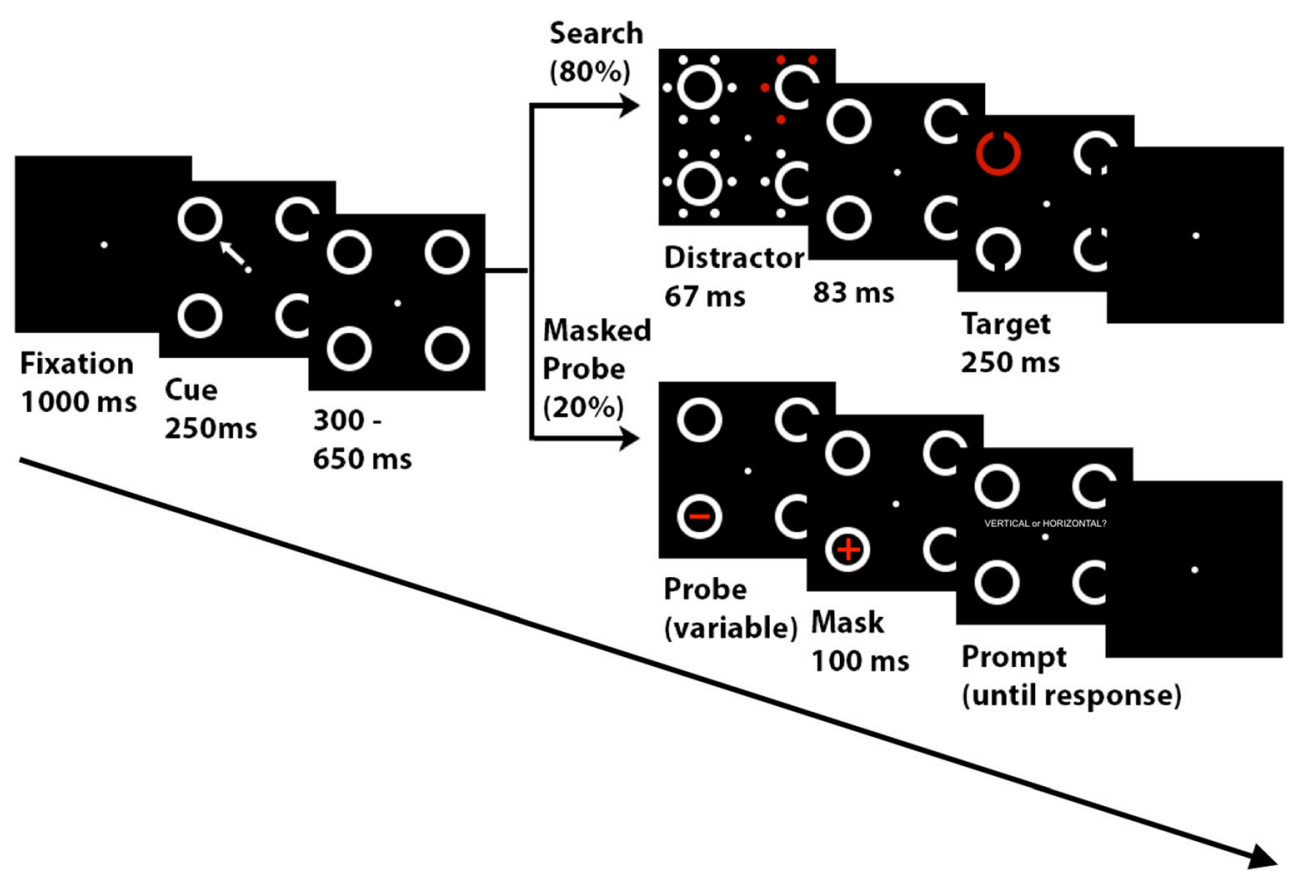

Fig. 6 Depiction of Experiment 2 trial events, including Search and Masked probe trials, which were mixed randomly within blocks. The variable probe exposure duration was titrated based on performance. See Materials and Methods for additional details

\section{Masked probe trials}

We analyzed probe response accuracy at three locations: cued location, uncued/predicted distractor location, and uncued/ non-predicted location. We collapsed across blocks because learning did not vary significantly by block in Experiment 1 and to maximize power, as probes were presented on only $20 \%$ of trials. As in Experiment 1, we excluded trials in which distractors were predicted at the cued location.

First, the manipulation of probe exposure duration succeeded in keeping participants' response accuracy within a sensitive range, from $64.1 \%$ to $81.2 \%$. Also, mean probe exposures did not vary across the three conditions specified above, $M=98 \mathrm{~ms}, 98 \mathrm{~ms}$, and $99 \mathrm{~ms} ; \mathrm{SD}=27 \mathrm{~ms}, 27 \mathrm{~ms}$, and $27 \mathrm{~ms}$, respectively.

For the critical pairwise comparison of this experiment, probes in the predicted distractor location yielded significantly poorer accuracy than in the non-predicted location, $t(23)=$ 2.20, $P=0.038, \eta_{p}{ }^{2}=0.174$ (Fig. 7). This result supports our hypothesis that implicitly learned distractor locations were suppressed; probe accuracy was also marginally greater at the cued location than the predicted distractor location, $t(23)=$ $1.92, P=0.066, \eta_{p}{ }^{2}=0.139$. Performance was numerically but not significantly greater at the cued location than the nonpredicted location, $t(23)=0.686$ (n.s.).

\section{Explicit learning assessment}

Nine participants reported noticing the distractor location was predictable and 15 did not. As in Experiment 1, neither group reliably predicted the location above chance, $t(8)=0.536$ (n.s.), and $t(14)=0.470$, (n.s.), respectively (Fig. 5).

\section{General discussion}

This study shows that individuals can implicitly learn to suppress spatial locations that are anticipated to contain salient, irrelevant stimuli. Moreover, this suppression can be implemented flexibly, on a trial-by-trial basis.

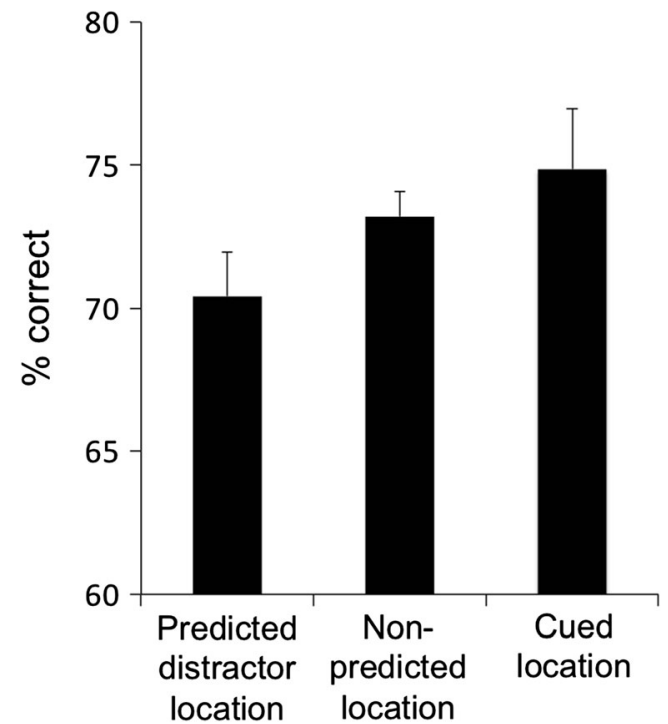

Fig. 7 Probe accuracy performance in Experiment 2. Error bars indicate SE of the mean 
One question frequently asked of studies claiming spatial suppression is whether such suppression can be established in advance (i.e., proactively), or if it instead reflects a more rapid, reactive disengagement (see Geng, 2014). Much like the impossibility of complying with a request to not think of a white bear, some have argued that trial-by-trial adjustments of spatial suppression can only be achieved reactively (Moher \& Egeth, 2012; Tsal \& Makovski, 2006). Note that distractors in those studies were defined based on features rather than locations (see Anderson \& Folk, 2012; Beck \& Hollingworth, 2015). Here, probe identification results from Experiment 2 are consistent with trial-by-trial shifts in proactive suppression. Our manipulation may have succeeded where others failed because of its implicit nature; that is, it should be easier to ignore a white bear when you are not consciously thinking about doing so.

One curiosity of our present data was the lack of a significant probe accuracy advantage to explicitly cued vs. uncued locations, suggesting that participants did not attentionally enhance the cued location. This seems especially puzzling given the RT advantage to cued locations during search. We can only offer speculation, but one possibility is that observers did not enhance cued target locations until after distractors appeared; thus at the moment of probe onset, target enhancement had yet to be implemented. Certainly these results call into question our assumptions about the temporal dynamics of attention in distraction paradigms like this, and we plan further experimentation to understand this intriguing result.

Additionally, we look forward to employing converging methods to further characterize the nature of implicitly learned suppression. Such methods include fMRI (e.g., Curtis et al., 2004; Ruff \& Driver, 2006), the distractor positivity ERP component (Sawaki \& Luck, 2010), or EEG alpha-band synchrony (Foxe \& Snyder, 2011; Payne, Guillory \& Sekuler, 2013).

To summarize, our results speak to the ecological validity and flexibility of spatial suppression. As we navigate familiar environments every day, we encounter various situations in which suppression would be behaviorally beneficial. A mechanism of suppression is indeed available, without the need for conscious knowledge or externally guided instructions.

Acknowledgments We thank Jessica Irons, Bo-Yeong Won, and two anonymous reviewers for helpful comments and suggestions. Support was provided by NSF BCS-1027054 and US-Israel BSF 2009424 to A. B. L.

\section{References}

Anderson, B. A., \& Folk, C. L. (2012). Dissociating location-specific inhibition and attention shifts: Evidence against the disengagement account of contingent capture. Attention, Perception, \& Psychophysics, 74(6), 1183-1198.

Beck, V. M., \& Hollingworth, A. (2015). Evidence for negative feature guidance in visual search is explained by spatial recoding. Journal of
Experimental Psychology: Human Perception and Performance, 41(5), 1190-1196.

Brainard, D. H. (1997). The psychophysics toolbox. Spatial Vision, 10, 433-436.

Chun, M. M., \& Jiang, Y. (1998). Contextual cueing: Implicit learning and memory of visual context guides spatial attention. Cognitive Psychology, 36(1), 28-71.

Curtis, C. E., Rao, V. Y., \& D’Esposito, M. (2004). Maintenance of spatial and motor codes during oculomotor delayed response tasks. Journal of Neuroscience, 24(16), 3944-3952.

Dhawan, S., Deubel, H., \& Jonikaitis, D. (2013). Inhibition of saccades elicits attentional suppression. Journal of Vision, 13(6), 9.

Eriksen, C. W., \& Collins, J. F. (1969). Temporal course of selective attention. Journal of Experimental Psychology, 80(2, Pt.1), 254 261.

Fiser, J., \& Aslin, R. N. (2001). Unsupervised statistical learning of higher-order spatial structures from visual scenes. Psychological Science, 12(6), 499-504.

Folk, C. L., Remington, R. W., \& Johnston, J. C. (1992). Involuntary covert orienting is contingent on attentional control settings. Journal of Experimental Psychology: Human Perception and Performance, 18(4), 1030-1044.

Foxe, J. J., \& Snyder, A. C. (2011). The role of alpha-band brain oscillations as a sensory suppression mechanism during selective attention. Frontiers in Psychology, 2, 154.

Geng, J. J. (2014). Attentional mechanisms of distractor suppression. Current Directions in Psychological Science, 23(2), 147-153.

Goschy, H., Bakos, S., Müller, H. J., \& Zehetleitner, M. (2014). Probability cueing of distractor locations: Both intertrial facilitation and statistical learning mediate interference reduction. Frontiers in Psychology, 5, 1195.

Hallett, P. E. (1978). Primary and secondary saccades to goals defined by instructions. Vision Research, 18(10), 1279-1296.

Kawahara, J. (2010). Identifying a "default" visual search mode with operant conditioning. Acta Psychologica, 135(1), 38-49.

Kelley, T. A., \& Yantis, S. (2009). Learning to attend: Effects of practice on information selection. Journal of Vision, 9(7), 16.

Klein, R. M. (2000). Inhibition of return. Trends in Cognitive Sciences, 4(4), 138-147.

Leber, A. B., Lechak, J. R., \& Tower-Richardi, S. M. (2013). What do fast response times tell us about attentional control? Journal of Vision, 13(3), 31.

Maljkovic, V., \& Nakayama, K. (1996). Priming of pop-out: II. The role of position. Perception \& Psychophysics, 58(7), 977-991.

Miller, J. (1988). Components of the location probability effect in visual search tasks. Journal of Experimental Psychology: Human Perception and Performance, 14(3), 453-471.

Moher, J., \& Egeth, H. E. (2012). The ignoring paradox: Cueing distractor features leads first to selection, then to inhibition of tobe-ignored items. Attention, Perception, \& Psychophysics, 74(8), $1590-1605$.

Munneke, J., Van der Stigchel, S., \& Theeuwes, J. (2008). Cueing the location of a distractor: An inhibitory mechanism of spatial attention? Acta Psychologica, 129(1), 101-107.

Munoz, D. P., \& Everling, S. (2004). Look away: The anti-saccade task and the voluntary control of eye movement. Nature Reviews Neuroscience, 5(3), 218-228.

Payne, L., Guillory, S., \& Sekuler, R. (2013). Attention-modulated alphaband oscillations protect against intrusion of irrelevant information. Journal of Cognitive Neuroscience, 25(9), 1463-1476.

Pelli, D. G. (1997). The VideoToolbox software for visual psychophysics: Transforming numbers into movies. Spatial Vision, 10(4), 437-442.

Posner, M. I., \& Cohen, Y. (1984). Components of visual orienting. Attention and Performance X: Control of Language Processes, 32, 531-556. 
Posner, M. I., Snyder, C. R., \& Davidson, B. J. (1980). Attention and the detection of signals. Journal of Experimental Psychology: General, $109(2), 160-174$

Proulx, M. J. (2011). Individual differences and metacognitive knowledge of visual search strategy. PLoS ONE, 6(10), e27043.

Reder, L. M., Weber, K., Shang, J., \& Vanyukov, P. M. (2003). The adaptive character of the attentional system: Statistical sensitivity in a target localization task. Journal of Experimental Psychology: Human Perception and Performance, 29(3), 631-649.

Ruff, C. C., \& Driver, J. (2006). Attentional preparation for a lateralized visual distractor: Behavioral and fMRI evidence. Journal of Cognitive Neuroscience, 18(4), 522-538.

Sawaki, R., \& Luck, S. J. (2010). Capture versus suppression of attention by salient singletons: electrophysiological evidence for an automatic attend-to-me signal. Attention, Perception, \& Psychophysics, 72(6), $1455-1470$.

Stadler, M. A., \& Frensch, P. A. (1998). Handbook of implicit learning. Thousand Oaks, CA: Sage.

Tsal, Y., \& Makovski, T. (2006). The attentional white bear phenomenon: The mandatory allocation of attention to expected distractor locations. Journal of Experimental Psychology: Human Perception and Performance, 32(2), 351-363.

Walthew, C., \& Gilchrist, I. D. (2006). Target location probability effects in visual search: An effect of sequential dependencies. Journal of Experimental Psychology: Human Perception and Performance, 32(5), 1294-1301.

Willingham, D. B., Nissen, M. J., \& Bullemer, P. (1989). On the development of procedural knowledge. Journal of Experimental Psychology: Learning, Memory, and Cognition, 15(6), 1047-1060. 